\title{
„Ich möchte den Dialog zwischen TePe und den Praxen fördern“
}

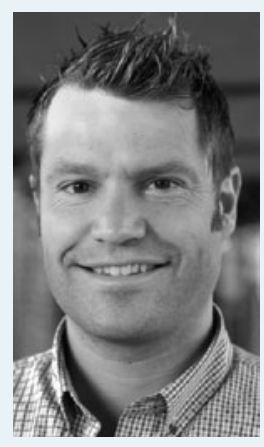

Zahnarzt Dr. Arne Wegner ist seit November 2013 in seiner Funktion als Dental Professional Specialist beim Mundhygienespezialisten TePe einer der Ansprechpartner des Unternehmens speziell für Behandler und das zahnmedizinische Fachpersonal.

? Welche Aufgaben nehmen Sie konkret bei TePe wahr?

Wegner: Ich bin Ansprechpartner für Mitarbeiter und Kunden bei fachlichen Fragen, unterstütze die Ausarbeitung von Schulungsvorträgen und Fachtexten und fördere die Vernetzung von TePe mit Experten aus Wissenschaft und Praxis. Gemeinsam mit anderen TePe-Mitarbeitern, die entweder als Zahnmediziner oder als Prophylaxefachkraft lange in Praxen gearbeitet haben, möchte ich so einen permanenten Dialog mit den Praxen fördern. Ich arbeite an der Schnittstelle zwischen den Herausforderungen der Praxis und der Möglichkeit, aktiv darauf $z u$ reagieren.

? Welchen Stellenwert nimmt TePe Ihrer Meinung nach für die Mundhygiene in Deutschland und darüberhinaus ein? Wegner: Die TePe-Interdentalbürsten sind in Deutschland marktführend. Zudem bietet TePe mit seinem Mundhygienesortiment über die Interdentalbürsten hinaus für jede intraorale Situation das passende Produkt. Damit sind alle Voraussetzungen für ein hohes Niveau der Mundhygiene geschaffen.

? Das Angebot an Mundhygieneprodukten ist groß. Wie sollte der Patient bei der Auswahl vorgehen?

Wegner: Nicht die Quantität der Mundhygieneprodukte im Zahnputzbecher, sondern die Qualität ihrer Anwendung zählt. Bekommt der Patient zu viele unterschiedliche Produkte empfohlen, kann die Putzmotivation und damit auch die Mundhygiene unter dem übermäßigen Umfang leiden. Am besten ist es sicherlich, wenn Patienten im Rahmen der fachkundigen
Beratung verschiedene Produkte ausprobieren können. Neben der Zahnbürste gehören zumindest 1 Produkt zur Interdentalpflege und in einigen Fällen auch eine Mundspülung zur Grundausstattung.

? Wie kann man eine präventiv orientierte Zahnheilkunde weiter fördern?

Wegner: Oberstes Gebot sollte immer sein, die Zähne möglichst lang zu erhalten. Dafür müssen Patienten zum einen intensiv und wiederholt über die Möglichkeiten der Prophylaxe für den Zahnerhalt aufgeklärt werden, damit sie die Notwendigkeit der häuslichen Zahnpflege nachvollziehen können. Zum anderen ist es natürlich wesentlich, dass geeignete Hilfsmittel für eine optimale Mundhygiene zur Verfügung stehen. Durch Fluoridierungsmaßnahmen kann bspw. eine Füllungstherapie im Anfangsstadium umgangen werden. Der Griff zum Bohrer ist also weniger schnell notwendig.

Nach einer Pressemitteilung der

TePe Mundhygieneprodukte Vertriebs-GmbH, Hamburg

Internet: www.tepe.com

\section{Erweiterung der Teilmatrizensysteme}

Mit Beginn des neuen Jahres hat DENTSPLY sein erfolgreiches Teilmatrizensystem Palodent Plus um die neuen Palodent Plus EZ Coat-Matrizen $(E Z=$,easy“) erweitert. Diese gut auf das Komplettsystem abgestimmten Matrizen besitzen eine mikrodünne AntihaftBeschichtung für noch einfacheres Entfernen sowie eine verbesserte Kurvatur für anatomisch korrekte Formen selbst bei besonders großen Kavitäten. Wie die Plus-Standardmatrizen sind auch die neuen Coat-Matrizen in 5 Größen für alle klinischen Situationen verfügbar. Eine übersichtliche Farbkodierung erleichtert die Größenauswahl: 3,5 mm (dunkelgrau), 4,5 mm (dunkelblau), 5,5 mm (mittelblau), 6,5 mm (hellblau) und $7,5 \mathrm{~mm}$ (hellgrau). Ergänzt wird das Teilmatrizensystem durch das Unterfüllungskomposit SDR (Smart Dentin Replacement). Die Kombination von Matrizen mit dem Komposit bietet zu-

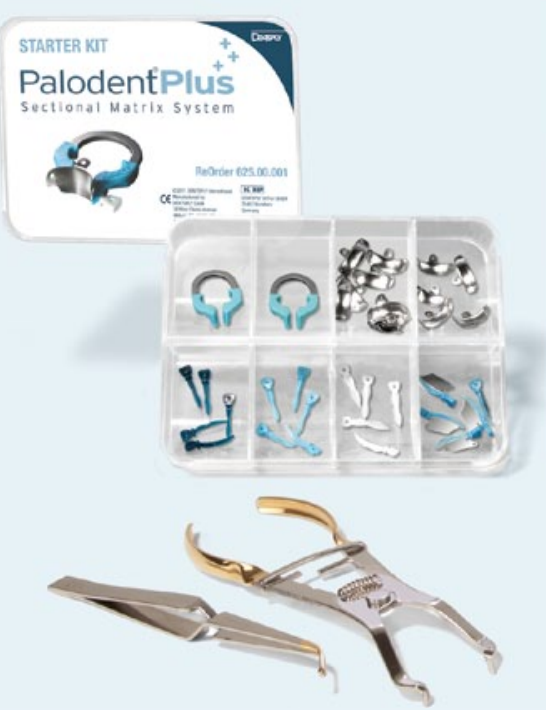

verlässige Klasse-II-Restaurationen mit dichten Kavitätenrändern und anatomisch korrekten Kontaktpunkten. Dank der dichten gingivalen Versiegelung gibt es weniger Überschuss und damit weniger Finieraufwand.

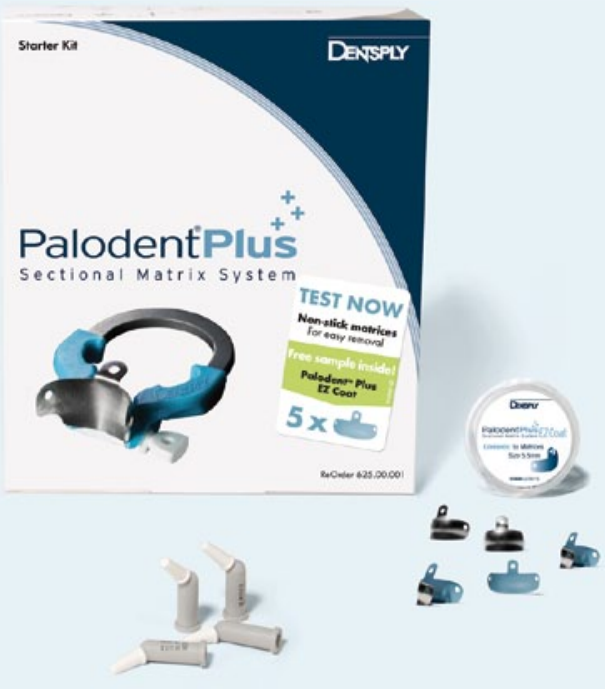

Weitere Informationen unter der gebührenfreien DENTSPLY Service-Line für Deutschland 08000-735000 sowie unter www.dentsply.de/palodentplus.

Nach einer Prssemitteilung der

Dentsply Detrey GmbH, Konstanz 
Röntgen

\section{D-Gerät mit niedrigster Patientendosis}

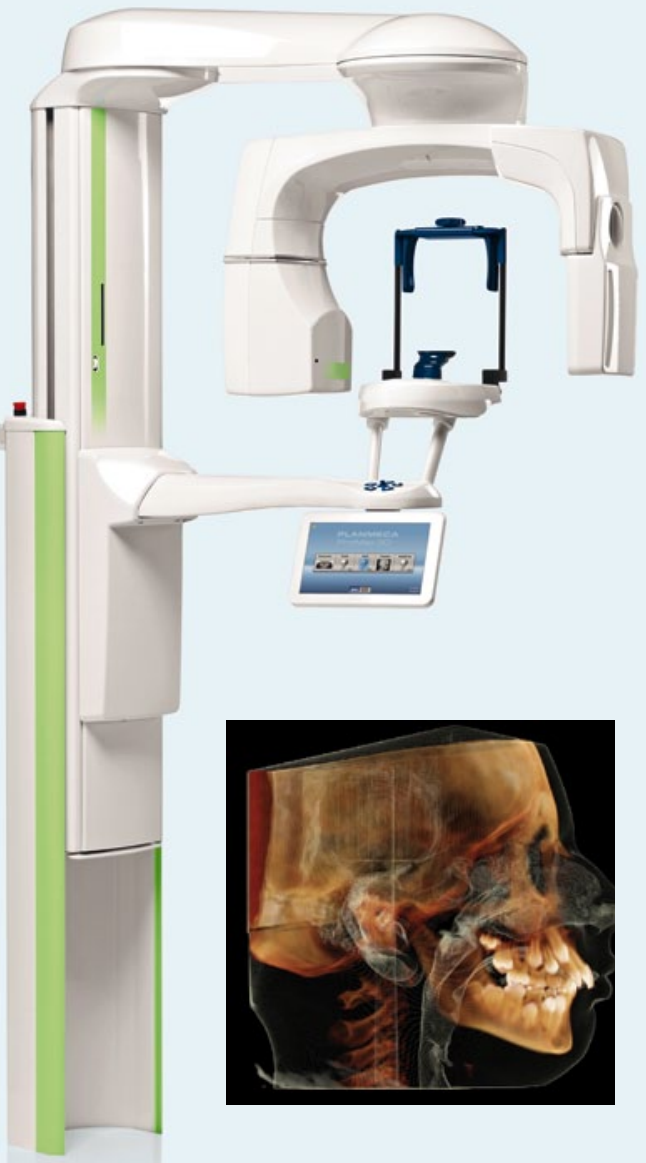

Planmeca hat den Ultra Low Dose-Modus entwickelt. Nur $14,4 \mu \mathrm{Sv}$ beträgt der niedrigste Wert der effektiven Patientendosis für eine 3D-Vollschädelaufnahme. Alle Planmeca ProMax-3DGeräte ermöglichen DVT-Bildgebung mit einer geringeren Strahlendosis als die traditionelle 2D-Panorama-Bildgebung. Trotz minimaler Strahlendosis werden anatomische Informationen detailliert dargestellt. Mit dem Ultra Low DoseModus sind zum Beispiel chirurgische Planungen, Verlaufskontrollen und die Lokalisation retinierter oder verlagerter Zähne möglich. Aber auch Gesichtsasymmetrien und kephalometrische Bezugspunkte können definiert werden. Selbst aussagefähige Sinus-Bildgebung oder Messungen der Luftwege in der HNO-Diagnostik sind machbar. Alle Planmeca ProMax-Röntgengeräte liefern eine Vielzahl an vorprogrammierten und leicht einstellbaren Bildgebungsmodalitäten für alle Volumengrößen.

Nach einer Pressemiteilung der

Planmeca Vertriebs $\mathrm{GmbH}$, Bielefeld

Internet: www.planmeca.com

Kostenlos

\section{Kompass für Vollkeramik-Restaurationen}

Keramisch denken, keramisch präparieren - nur so können hochwertige, langlebige vollkeramische Restaurationen entstehen. Um alle Präparation- und Bearbeitungsregeln schnell und einfach „zur Hand“ zu haben, hat Komet ein praktisches kleines Ringbuch entworfen. Dieser kostenlose Kompass führt auf 20 Seiten durch die keramikgerechte Präparation und Bearbeitung. Präparationsempfehlungen gehen dabei prak-

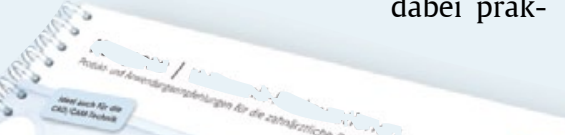

tischerweise Hand in Hand mit der Vorstellung diverser Instrumente bzw. Sets, die Komet rund um die Präparation und Bearbeitung von Keramik speziell entwickelt hat. Dazu zählen die beiden Experten-Sets 4562ST (für Keramik-Inlays und Teilkronen) und 4573ST (für KeramikKronen), die diamantierten Schallspitzen SFM7 und SFD7 für die approximale Kavitätenpräparation von Prämolaren und Molaren, die Sets 4637 und 4622 für die souveräne Bearbeitung/Anpassung von Zirkonoxid sowie der Kronentrenner 4ZR. Damit bietet der neue Kompass Zahnärzten die Chance, korrekt und souverän mit Keramik umzugehen: Fordern Sie ihn an, unkompliziert und kostenlos!

Nach einer Pressmitteilung der Komet Dental Gebr. Brasseler

GmbH \& Co. KG, Lemgo Internet: www.kometdental.de
Endodontie

Neue TorqTech-

Winkelstücke ergänzen Produktpalette

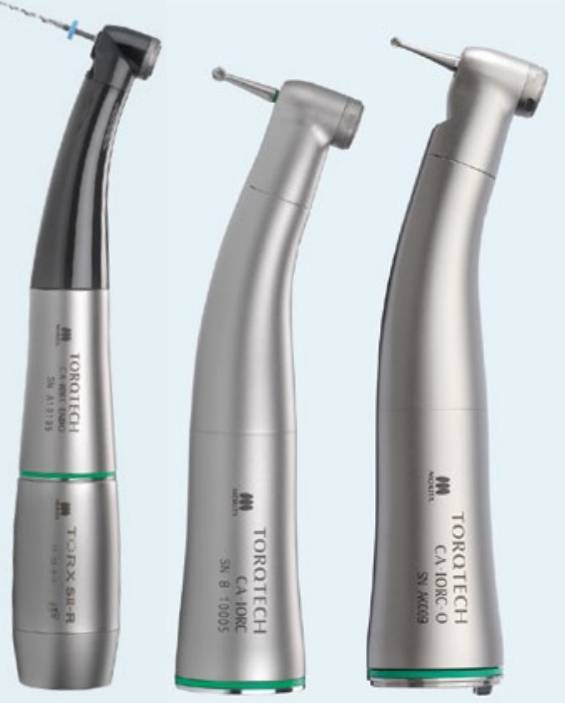

Die TorqTech Hand- und Winkelstücke von Morita sind ergonomisch geformt, kompakt in den Abmessungen und mit sehr kleinen Instrumentenköpfen ausgestattet. Ergänzt wurde diese Produktreihe jüngst durch das neue Winkelstück TorqTech CA-10RC bzw. CA-10RC-O. TorqTech CA-10RC wurde für die Anwendung bei der maschinellen Wurzelkanalaufbereitung mit Instrumenten aus NickelTitan konzipiert. Mit einem Gewicht von nur $67 \mathrm{~g}$ liegt das Winkelstück CA-10RC extrem leicht in der Hand, während sein Pendant CA-10RC-O mit nur $2 \mathrm{~g}$ mehr aufwartet. Dafür ist in diesem Winkelstück ein Lichtleiter eingebaut, während das CA-10RC ohne Lichtquelle auskommt. Zahnärzte profitieren von dem geringen Durchmesser des Instrumentenkopfes mit nur $8 \mathrm{~mm}$. Weiterhin gemeinsam ist beiden Winkelstücken wie auch den anderen Hand- und Winkelstücken der TorqTech-Serie das Merkmal der glatten, keramikbeschichteten Oberfläche. Nur für die Premium-Behandlungseinheit Soaric von Morita steht das Winkelstück CA-10RC-ENDO zur Verfügung - mit diesem Endowinkelstück kann die Wurzelkanalaufbereitung mit Instrumenten aus Nickel-Titan unter permanenter Längenkontrolle des integrierten Apex-Lokators sicher durchgeführt werden.

Nach einer Pressemitteilung J. Morita Europe GmbH, Dietzenbach Internet: www.morita.com/europe 
Implantatprothetik

\section{Verschraubt vs.}

\section{zementiert}

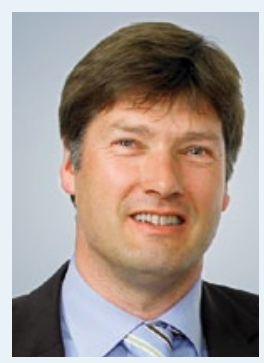

Fragen an

Thomas Schwarz,

Global Business

Development

Manager Digital

bei Heraeus

Kulzer, zur

Suprastruktur

cara I-Bridge.

? Suprastrukturen zu verschrauben ist nicht neu. Ist der Trend zum Schrauben nicht ein Rückschritt?

Schwarz: Heute ist die technische Entwicklung viel weiter. Die Hersteller haben die Schwächen von damals behoben, zum Beispiel durch die Verwendung anderer Materialien und die präzise CAD/ CAM-Fertigung der Suprastrukturen. Andere Länder sind Deutschland voraus. Doch der Trend zurück zur verschraubten Lösung kommt auch langsam in Deutschland wieder an. Großanwender, die viele Implantate setzen, haben verschraubte Lösungen für sich entdeckt, da sie ihnen eine höhere Sicherheit und Wirtschaftlichkeit bieten.

\section{? Lohnt sich ein Wechsel zur cara I-Bridge wirtschaftlich?}

Schwarz: Ja! Der Wechsel lohnt allein, da der Zahnarzt mit nur einem Schraubenzieher arbeitet, egal auf welchem Implantatsystem. Das spart Zeit und den Kauf vieler Werkzeuge.

\section{? Stichwort Periimplantitis: Wie} sieht das bei verschraubten Lösungen aus?

Schwarz: Hygienisch liegt ein Vorteil verschraubter Arbeiten im einfachen Herausnehmen und Wiedereinsetzen. Von dieser Möglichkeit sollten Anwender Gebrauch machen, um das Risiko einer Periimplantitis so gering wie möglich zu halten. Entscheidend ist, die Patienten durch Beratung und Service in der Hygiene zu unterstützen. Bei verschraubten Brücken kann der Zahnarzt zum Beispiel einen jährlichen Recall anbieten, um die Brücke zu prüfen und zu reinigen.

Nach einer Pressemitteilung der Heraeus Kulzer GmbH, Hanau Internet: www.heraeus-kulzer.com
Mikromotor

\section{Leicht, handlich und überall einsetzbar}

Der neue Mikromotor Bravo Marathon Portable III, aus dem Hause Hager \& Werken, ist ein durchzugsstarker, tragbarer, schnurloser Mikromotor, der sich platzsparend am Gürtel (Gürteltasche im Lieferumfang enthalten) des Behandlers anbringen lässt. Er ermöglicht das Arbeiten ohne Steckdose und ist damit ideal für den Hausbesuch geeignet, um z.B. eine Druckstelle an einer Prothese zu beheben. Der Motor verfügt über einen leisen, stufenlos regelbareren Motor mit Vor- und Rückwärtslauf (4000-35000 U/min), sowie einen Lithium-Akku für eine gesteigerte Laufzeit von ca. $12 \mathrm{~h}$ (über $30 \%$ mehr Laufzeit als sein Vorgängermodell) bei einer geringen Ladedauer von nur $5 \mathrm{~h}$ und einer Batterielebensdauer von über 500 Ladungen. Er ist geeignet für jedes Standardhand- und -winkelstück mit planem Aufnahmeschaft (ohne Intraspray und ohne Lichtleiter) und ist nur für den extraoralen Bereich verwendbar.
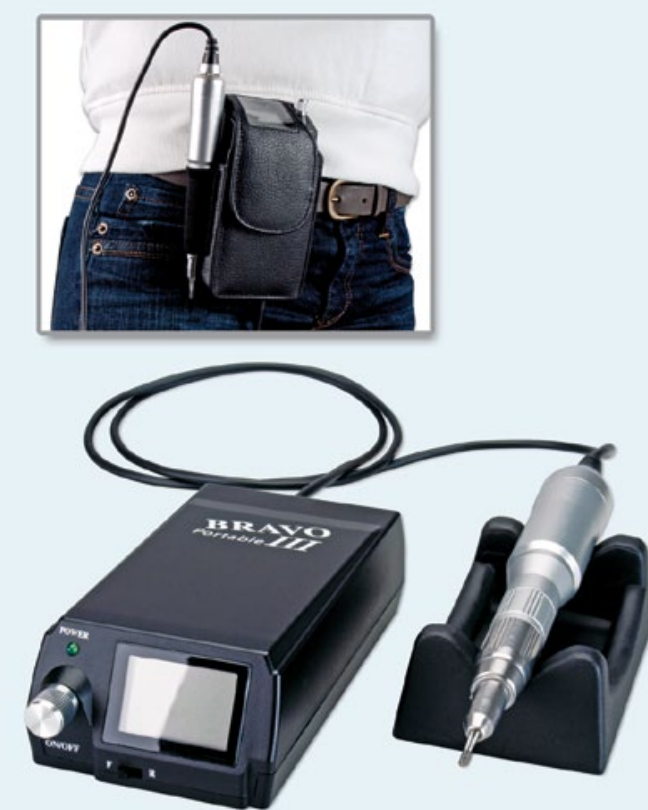

Nach einer Pressemitteilung der Hager \& Werken GmbH \& Co. KG, Duisburg Internet: www.hagerwerken.de

\section{Fortbildungsprogramm 2014 \\ Fortbildungen sind mobil per App verfügbar}

DENTSPLY Implants präsentiert das gesamte Fortbildungsprogramm 2014 in einer innovativen Smartphone-App. Die neue App steht momentan für iPhone, iPad und iPod touch zur Verfügung und ermöglicht eine gezielte individuelle Suche nach Fachgebiet, Produktsystem, Ort, Zeitraum, Referenten oder Zielgruppe. Alle wichtigen Details sind auf einer Seite sichtbar. Nach einem kurzen Fingertippen wird eine ausführliche Beschreibung des Fortbildungsinhalts angezeigt, und der Kursflyer kann heruntergeladen werden. Im Bereich „Anmeldung“ gibt es die Möglichkeit, sich online zu registrieren, bei Rückfragen eine E-Mail an das Veranstaltungsteam zu senden oder auch direkt dort anzurufen. Wer gerne zusammen mit einer Kollegin oder einem Kollegen eine Fortbildung besuchen möchte, kann über einen WeiterleitungsLink auf die präferierte Fortbildung aufmerksam machen. Ein individueller Merkzettel hilft dem Interessenten, stets einen Überblick über die geplanten oder bereits gebuchten Veranstaltungen zu behalten. Der Termin kann automatisch

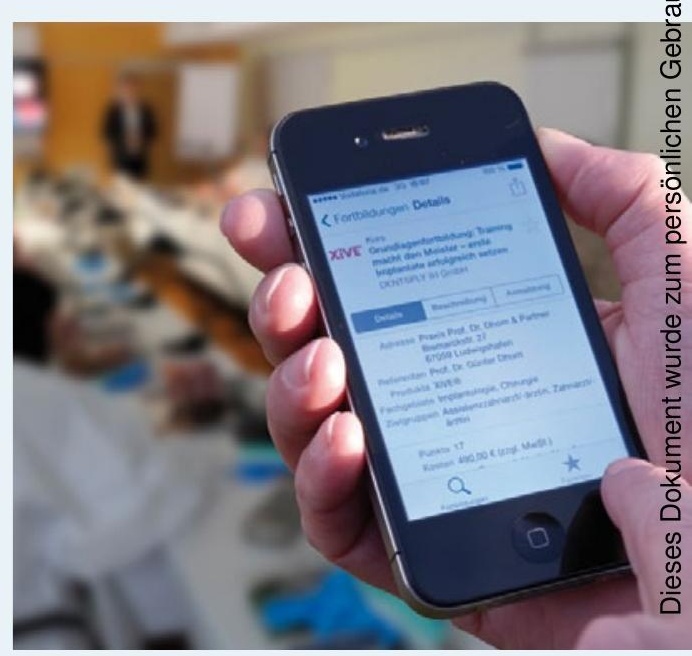

in den Kalender übertragen werden. Die integrierte Kartenfunktion führt den Teilnehmer direkt zum Ort der Veranstaltung. Alle Suchfunktionen der App sowie die Online-Anmeldung sind auch auf der Fortbildungs-Webseite von DENTSPLY Implants verfügbar: www.dentsplyimplants.de/Fortbildung.

Nach einer Pressemitteilung der DENTSPLY IH GmbH, Mannheim 


\section{Neue CMD-Software fusioniert erstmals DVT- und JMT-Daten}

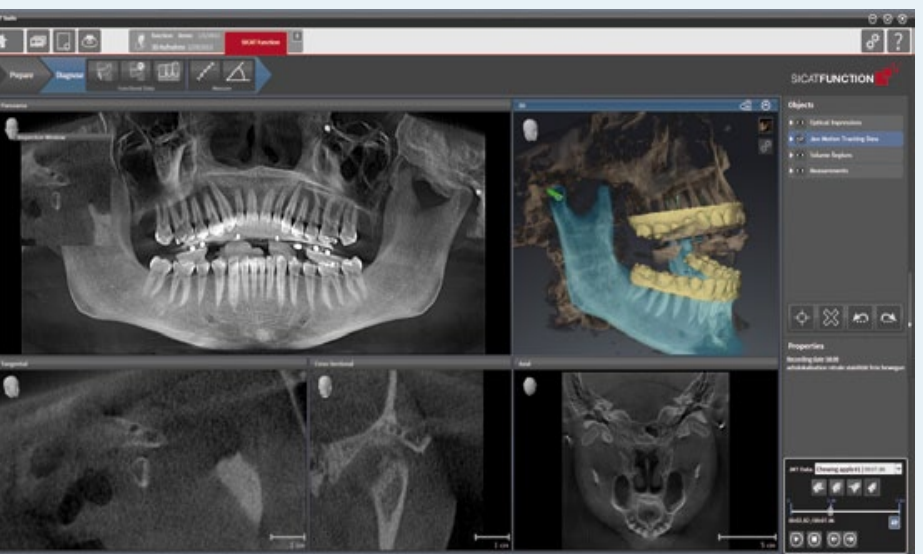

che diagnostische Patienteninformationen des DVT's (GALILEOS, Sirona)/CT's, eines Jaw Motion Trackers (SICAT JMT+) und optische Abdrücke (CEREC, Sirona) präzise fusioniert. So kann die echte patientenindividuelle Bewegung des Unterkiefers im 3D-Volumen anatomiegetreu dargestellt werden. Die räumliche Beziehung SICAT präsentiert jetzt das Markt-Release von SICAT Function, einer Lösung, wel- von Kondylus und Fossa während der Bewegung ist individuell darstellbar. An jedem spezifischen Punkt des Kondylus und der Mandibula lassen sich die Bewegungsspuren visualisieren - und dies je nach Präferenz des Behandlers. Auch die dynamische Okklusion kann anhand der optischen Abdrücke für jede Kieferstellung nachvollzogen werden. Bei Vorliegen aller relevanten Patientendaten sind deshalb Diagnose, Planung und Beratung mit dem Patienten in einer Sitzung möglich. Je nach Präferenz des Behandlers kann dann eine entsprechende MichiganBehandlungsschiene bei SICAT bestellt werden.

Nach einer Pressemitteilung der

SICAT GmbH \& Co. KG, Bonn

Internet: www.sicat.de

\section{Effektive und langanhaltende Wirksamkeit bei dentiner Hypersensibilitåt}

Für Patienten mit hypersensitiven Zähnen ist mit dem neuen Listerine Professional Sensitiv-Therapie das 1. Listerine-Therapeutikum für schmerzempfindliche Zähne entwickelt worden. Die patentierte Behandlung mit KristallTechnologie bekämpft die Ursache des Schmerzes, offene Dentinkanäle. Das in der Mundspülung enthaltene Oxalat bildet in Verbindung mit Speichel Kristalle, die sich nicht nur auf der Denti- noberfläche, sondern auch tief in den offenen Dentinkanälen ablagern. Dadurch entsteht ein stabiler, physikalischer Schutzschild für die freiliegenden Nerven. Dank der patentierten KristallTechnologie verschließt die Mundspülung bereits nach 6 Spülungen 92\% der offenen Dentinkanälchen, nach 9 Spülungen bereits $100 \%$. Ab April 2014 steht Patienten mit dentiner Hypersensibilität mit der Mundspülung, die ausschließ-

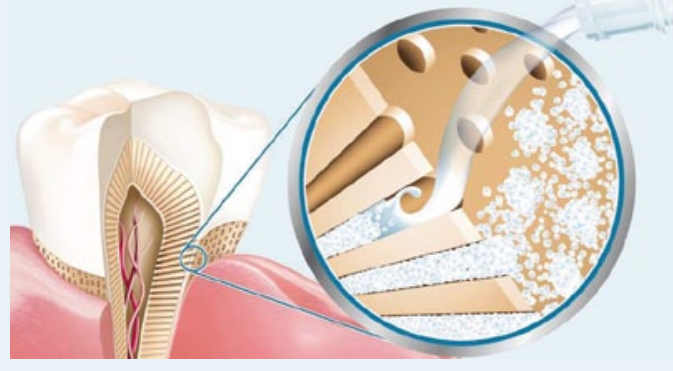

lich in der Apotheke erhältlich ist, eine effektive Behandlung zur regelmäßigen Anwendung zur Verfügung.

Nach einer Pressmitteilung der Johnson \& Johnson GmbH, Neuss

\section{Angebot der Lokalanåsthetika durch Injektionskanülen komplettiert}

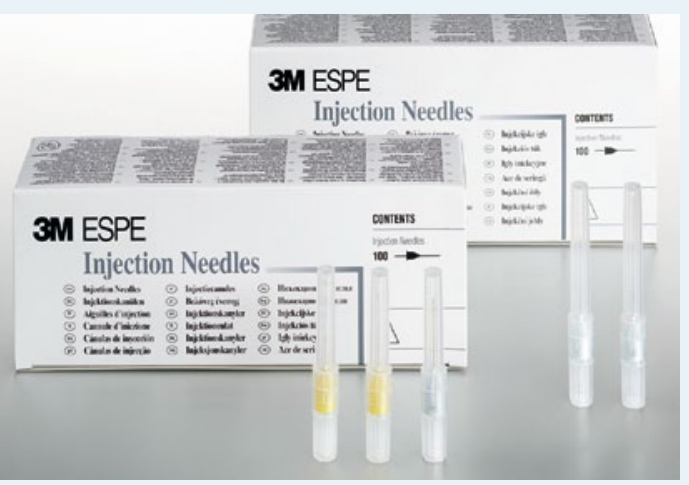

Um das Angebot an Lösungen für die Lokalanästhesie zu vervollständigen, führt 3M ESPE im März 2014 ein neues Produkt ein: 3M ESPE-Injektionskanülen. Diese sind in 5 verschiedenen Größen erhältlich und werden von dem deutschen Unternehmen Transcodent unter Einhaltung modernster Qualitätsstandards produziert. Dank ihrer besonderen Eigenschaften, die unter anderem einen für den Patienten angenehmeren Einstich ermöglichen und so $\mathrm{zu}$ einem erhöhten Patientenkomfort beitragen, erhielten die Kanülen eine sehr gute Bewertung von
4,5 Pluszeichen von der unabhängigen Publikation THE DENTAL ADVISOR (www. dentaladvisor.com). Die 5 Größen: 2 lange $(0,4 \times 42 \mathrm{~mm}$ und $0,4 \times 30 \mathrm{~mm}), 2$ kurze $(0,4 \times 25 \mathrm{~mm}$ und $0,3 \times 25 \mathrm{~mm})$ und 1 extrakurze $(0,3 \times 12 \mathrm{~mm})$ Variante. Die Kanülen runden das bestehende Angebot der Lokalanästhetika von 3M ESPE optimal ab. Dieses besteht u.a. aus Ubistesin (Wirkstoff Articain) und Mepivastesin (Wirkstoff Mepivacain). Die neuen 3M ESPE Injektionskanülen sind ab März 2014 erhältlich.

Nach einer Pressemitteilung der 3M Deutschland GmbH, Seefeld 